\title{
Gilles Kepel
}

Avec la collaboration de Leyla Arslan, Sarah Zouheir

\section{Banlieue \\ de la République}

Résumé

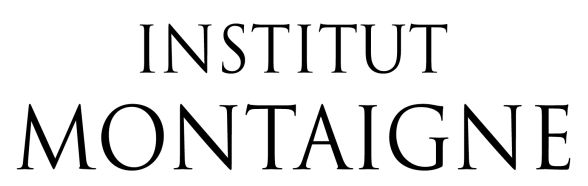

(14)

www.banlieue-de-la-republique.fr

www.institutmontaigne.org

Octobre 2011 


\section{Sommaire}

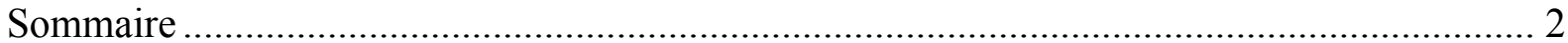

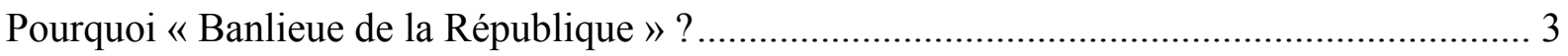

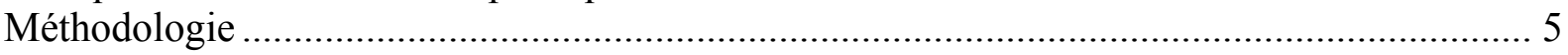

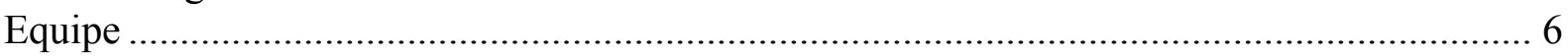

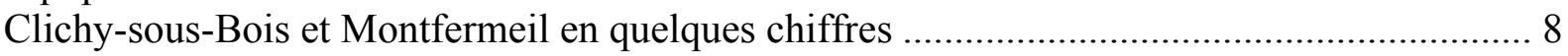

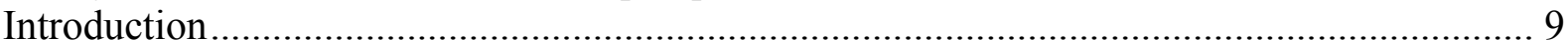

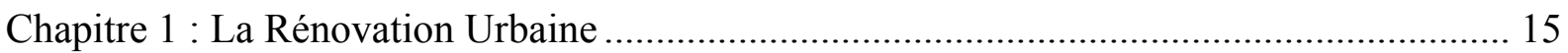

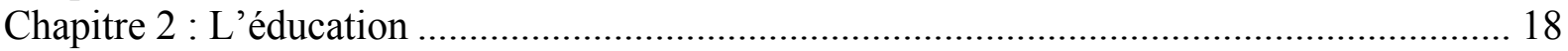

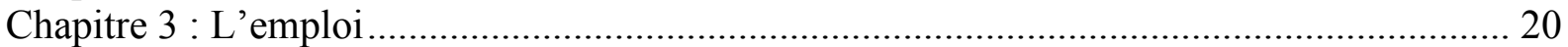

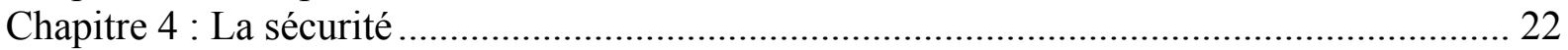

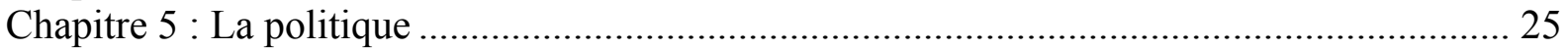

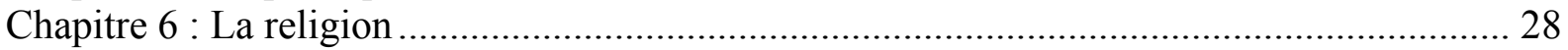

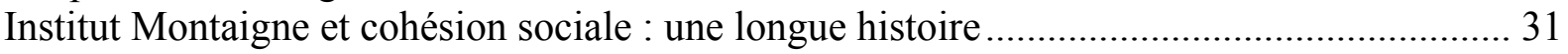

L'Institut Montaigne travaille depuis dix ans sur l'établissement de propositions concrètes au service de la compétitivité de notre pays et de sa cohésion sociale. L'enquête résumée ici est un document inédit sur l'agglomération de Clichy-Montfermeil, mais elle nous livre également de très nombreux enseignements sur le fonctionnement des quartiers en relégation et des zones urbaines sensibles.

Ce travail sera très prochainement publié sous forme de livre afin de le rendre accessible au plus grand nombre. Vous pouvez également retrouver les principaux apports de cette enquête sur le web.

Des propositions concrètes de l'Institut Montaigne, ainsi que des débats publics permettront d'aller plus loin durant toute la campagne pour l'élection présidentielle. Suivez-nous sur www.banlieue-de-larepublique.fr et www.institutmontaigne.org 


\section{Pourquoi « Banlieue de la République » ?}

Plusieurs décennies ont passé depuis que les enjeux de politique urbaine se sont invités au cœur de notre débat public, poussant parfois notre communauté nationale jusqu'au traumatisme comme à l'occasion des émeutes de 2005. Chaque élection nationale est désormais alimentée par son flot d'instrumentalisation et de propositions pour une amélioration de la vie dans ces quartiers. Beaucoup a été tenté et entrepris. Par exemple, le programme national de rénovation urbaine décidé en 2003 a mobilisé plus de 40 milliards d'euros, dont 600 millions d'euros pour la seule agglomération de Clichy-sous-Bois et Montfermeil, en Seine-Saint-Denis, épicentre des émeutes de 2005. Au total, ce programme entend améliorer le quotidien de quatre millions de nos concitoyens.

Les rapports de toute sorte n'ont pas manqué - et l'Institut Montaigne y a pris sa part -, ainsi que les créations institutionnelles parfois les plus originales. Aux yeux des Français, cette mobilisation n'a pas vraiment porté ses fruits et les résultats restent décevants. Par endroit, la situation économique, sociale et politique s'est tellement dégradée, que personne ne voit plus vraiment comment mettre fin à des logiques d'enfermement et de communautarisation. Il y a fort à parier que les échéances électorales à venir risquent à nouveau de révéler une abstention électorale massive et une implantation renforcée des extrêmes dans ces quartiers.

Difficulté supplémentaire, le télescopage des problèmes structurels auxquels est confronté notre pays à commencer par la capacité de son modèle économique à affronter la compétition du monde globalisé et à raviver la cohésion d'une société malade du chômage de sa jeunesse - avec la panne de notre modèle d'intégration nationale semble paralyser nos dirigeants politiques. Coincés entre immigration, islam, identité nationale et insécurité, ils ne parviennent plus à tracer un cap fédérateur et porteur de sens pour une majorité de Français.

Pour mieux comprendre ce qui se joue dans ces territoires, nous avons souhaité mener pendant plus d'un an une enquête en profondeur, précisément à Clichy-sous-Bois et Montfermeil. Conduite auprès des habitants, en français, mais aussi en arabe, en turc, en cambodgien, en anglais, en peulh, en soninké, elle s'intéresse à l'ensemble des dimensions qui permettent de " faire société »: le logement et la rénovation urbaine, l'éducation, l'emploi, la sécurité. Elle se termine en se demandant à quel point ces dimensions influencent le rapport des personnes interrogées au fait politique et aux questions religieuses. Confiée à Gilles Kepel, universitaire reconnu et auteur d'une première enquête sur la naissance de l'islam en France conduite en 1985, ainsi qu'à une équipe de chercheurs, cette monographie d'un territoire qui a été le foyer des émeutes urbaines de 2005 , est à nos yeux un document exceptionnel et sans équivalent à ce jour.

Bien entendu, cette enquête ne prétend pas fonder un diagnostic général sur la situation des quartiers et banlieues en France : Clichy et Montfermeil présentent des caractéristiques qui leur sont propres et qui ne sont pas toutes généralisables. Néanmoins, elle invite à une réflexion nécessaire.

Que dit l'enquête ? Tout d'abord que la rénovation urbaine a bien eu lieu et qu'elle est perceptible par les habitants des quartiers concernés. Mais elle dit aussi qu'à elle seule la rénovation du bâti ne peut suffire à assurer cohésion et développement dans des quartiers qui concentrent un grand nombre de difficultés. Pour être efficace, elle doit être accompagnée de politiques encore plus ambitieuses en matière d'éducation, d'emploi, de santé, de transports, de sécurité, et que ces politiques doivent être concertées.

Agir avec force et conviction est un impératif. Aux yeux de certains c'est un impératif moral, tant la situation vécue par les populations des quartiers relégués est parfois dramatique. Mais c'est aussi un impératif politique et économique, une exigence de bon sens pourrait-on dire : une partie de notre avenir se joue dans ces quartiers qui concentrent une population jeune très nombreuse. L'inquiétude des interlocuteurs de Gilles Kepel et de son équipe sur l'éducation, la civilité des enfants et des jeunes 
pose des problèmes fondamentaux sur l'éducation en banlieue.

L'action des pouvoirs publics dans ces quartiers populaires est évidemment déterminante. La politique de la ville est souvent critiquée pour son manque d'efficacité tant il est vrai qu'une politique publique d'exception ne peut rien si elle n'est pas fortement articulée aux politiques de droit commun. Mais on ne peut tout attendre de la puissance publique et des collectivités locales. Pour changer les choses de façon durable, d'autres acteurs ont un rôle majeur à jouer. C'est le cas en particulier des citoyens, des entreprises qui recrutent et des associations qui animent la vie de ces territoires tout en créant du lien social.

Sans doute est-ce la faible capacité d'attraction de la promesse laïque et républicaine qui interrogera le plus les lecteurs de ce travail. Il faudrait, pour porter remède à cette situation, que l'insertion dans la société par l'emploi rende aux habitants de ces quartiers dans leur diversité une pleine croyance dans les valeurs de la Nation, et que l'éducation leur en ait fourni les capacités, face aux défis quotidiens d'un environnement où l'ensemble de nos repères communs évolue sans cesse.

Mais rien d'efficace ne se fera sans une compréhension profonde d'un problème si complexe. C'est elle que cette enquête est allée chercher et c'est ce qui permettra d'avancer des propositions utiles dans le débat public.

Claude Bébéar, Nicolas Baverez, Jacques Bentz, Guy Carcassonne, Mireille Faugère, Christian Forestier, Michel Godet, Françoise Holder, Henri Lachmann, Bernard de La Rochefoucauld, Natalie Rastoin, Jean-Paul Tran Thiet, Arnaud Vaissié, Philippe Wahl et Lionel Zinsou sont membres du Comité directeur de l'Institut Montaigne, qui vient de publier "Banlieue de la République ». 


\section{Méthodologie}

Suite aux violences urbaines de l'automne 2005, de nombreuses interrogations sont nées sur la capacité française de faire société. Ce malaise est-il à mettre sur le compte d'une panne du modèle d'intégration culturelle ou sur des problèmes d'insertion sociale plus larges allant au-delà de la seule question des « immigrés »? Dans la lignée de ses travaux sur la société française, l'Institut Montaigne s'est penche sur ces interrogations et a mené, avec une équipe de recherche, une enquête inédite d'un an (juillet 2010-juin 2011) sur le territoire où se sont déclenchées les émeutes: la communauté d'agglomération de Clichy-sous-Bois/Montfermeil en Seine-Saint-Denis.

Cette enquête en profondeur menée par l'Institut Montaigne s'intéresse à différentes thématiques afin de comprendre les dynamiques à l'œuvre dans ce type de territoire : logement et rénovation urbaine, éducation, emploi, sécurité, politique et questions religieuses.

Dans un premier temps, une enquête auprès de 100 habitants de l'agglomération a été réalisée pendant un an, notamment auprès de Français et d'étrangers (régularisés ou non). La composition de l'échantillon n'a pas pour but d'être représentative statistiquement de la population de la communauté d'agglomération. Elle s'est plutôt attachée à mettre lumière sa diversité (selon des critères d'âge, de sexe, de religion, de classe sociale, de niveau d'études et de catégories socioprofessionnelles, de sousensemble d'habitation). Les entretiens ont été conduits en français, en anglais, en arabe, en turc, en peuhl, en soninké et en cambodgien

Pour protéger l'anonymat des enquêtés qui ont accepté de collaborer à cette recherche, les prénoms, noms et surnoms ont été changés. 


\section{Equipe}

\section{Gilles Kepel}

Né en 1955, docteur en science politique et en sociologie, Gilles Kepel est Professeur à l'Institut d'Etudes Politiques de Paris depuis 2001 et membre senior de l'Institut universitaire de France depuis 2010. Il est également senior fellow à la London School of Economics. Arabisant, Gilles Kepel est spécialiste du monde arabe et de l'islam. Entre 1980 et 2001, il a été successivement chercheur au Centre d'études juridiques, économiques et sociales (CEDEJ) du Caire, au CNRS, à la New York University et à Columbia University. Il a publié de nombreux ouvrages sur les mouvements islamistes, traduits en plusieurs langues, dont notamment : Le Prophète et Pharaon, aux sources des mouvements islamistes (1984), La Revanche de Dieu: Chrétiens, juifs et musulmans à la reconquête du monde (1991, réédité en 2003), Jihad, expansion et déclin de l'Islamisme (2000). Il fut l'un des premiers chercheurs français à étudier les musulmans en France à travers son ouvrage Les banlieues de l'Islam, naissance d'une religion en France, publié en 1987.

\section{Leyla Arslan}

Docteure en science politique, arabisante, elle travaille sur les quartiers populaires et les questions d'immigration et d'islam. Sa thèse a été publiée aux Presses universitaires de France (PUF) en 2011 sous le titre Enfants d'Islam et de Marianne : des banlieues à l'université. Elle interroge le rôle de l'ascension sociale permise notamment par l'école dans la construction de l'ethnicité et du religieux ainsi que de son déploiement dans la sphère publique et privée. Elle est également l'auteur de nombreux articles.

\section{Sarah Zouheir}

Diplômée de l'Institut d'Etudes Politiques de Paris et de la London School of Economics en politiques urbaines, elle s'intéresse aux politiques de logement, au développement économique local et à la gouvernance métropolitaine. Elle a participé à une étude sur les "housing associations 》 pour le Walterton and Elgin Community Homes à Londres.

\section{Mohamed-Ali Adraoui}

Chercheur et chargé de cours à l'Institut d'Etudes Politiques de Paris. Diplômé en économie, en sociologie politique et en relations internationales, sa thèse porte sur le salafisme en France. Il enseigne la politique dans le monde arabe. Ses publications portent sur l'islam. Auteur du rapport pour le ministère de l'Intérieur sur le salafisme, il a également écrit dans les ouvrages de référence Qu'estce que le salafisme? et Global Salafism. Islam's New Religious Movement. Il intervient régulièrement dans les médias français et internationaux. Il s'intéresse également aux changements récents dans le monde arabe et au conflit au Proche-Orient.

\section{Dilek Yankaya}

Docteure en science politique, elle a travaillé sur la nouvelle bourgeoisie islamique en Turquie. Au croisement de la science politique et de la sociologie, cette thèse étudie la consolidation politique, économique et ethico-culturelle du nouveau groupe de chefs d'entreprise musulmans pieux. Elle est en 
cours de publication aux Presses universitaires de France pour 2012. Chercheuse et consultante, les domaines d'étude de Dilek Yankaya concernent les problématiques liées à la production de nouvelles " manières d'être » islamique dans l'ère de la globalisation économique. Son expertise porte sur l'intégration de la culture islamique dans les stratégies d'investissement et de financement des entrepreneurs musulmans.

\section{Antoine Jardin}

Après des études d'histoire et de sociologie, il a entamé un travail de recherche sur le vote et l'abstention en banlieue dans le cadre d'une thèse de doctorat au Centre d'Etudes Européennes de l'Institut d'Etudes Politiques de Paris. Ses travaux soutenus par la région Île-de-France portent sur la dimension spatiale des inégalités en relation avec les attitudes et comportements politiques. 


\section{Clichy-sous-Bois et Montfermeil en quelques chiffres}

60000 habitants dans l'agglomération

Taux de chômage :

- Clichy-sous-Bois : 22,7\%

- Montfermeil : 17,5\%

- Ile de France : $11 \%$

Poids des moins de 14 ans dans la population totale :

- Clichy-sous-Bois : 28,55\%

- Montfermeil : 22,5\%

- Ile de France : 19,5\%

Part des foyers non imposables :

- Clichy-sous-Bois : $61,30 \%$

- Montfermeil : 45,40\%

- Ile de France : 33,60 \%

Pourcentage de la population de nationalité étrangère :

- Clichy-sous-Bois : $33 \%$

- Montfermeil : $20 \%$

- Ile de France : $12,4 \%$

Part des mineurs ayant au moins un parent né à l'étranger :

- Clichy-sous-Bois : $76 \%$

- Montfermeil : $50 \%$

- Ile de France : $16,9 \%$ 


\section{Introduction}

Au printemps 2010, l'Institut Montaigne me proposa de mener une étude sur l'articulation entre société, politique et religion en banlieue, autant de questions qui s'imposaient de manière aussi récurrente que confuse au coeur du débat national français. Immigration, islam, identité nationale, insécurité : le télescopage de ces quatre « $\mathrm{i}$ » avec les problèmes structurels auxquels est confronté notre pays - à commencer par la capacité de son modèle économique à affronter la compétition du monde globalisé et à raviver la cohésion d'une société malade du chômage structurel de sa jeunesse brouillait la réflexion publique plus qu'il ne la clarifiait.

Un quart de siècle auparavant, à l'été 1985, j'avais mené une première enquête sur la naissance de l'islam en France. Il s'agissait à l'époque de décrire un phénomène inédit, pour en interroger l'inscription présente et future dans le paysage national. Ce travail d'un trop jeune chercheur était resté sans grande incidence sur la réflexion publique, mais notre société fut interpellée avec réitération au long des vingt-cinq dernières années par les développements complexes de ce qui était devenu l'islam de France. La présente étude, si elle fait écho à la première, s'en distingue à la fois par l'objectif et le contenu. En liaison avec l'Institut qui l'a commanditée, elle voudrait en effet contribuer avec d'autres au dialogue sur la société française et ses valeurs dont la campagne pour l'élection présidentielle de 2012 ne peut faire l'économie - même si ce travail aspire aussi à poser des jalons pour les débats ultérieurs. Et elle ne porte pas sur l'émergence de l'islam comme tel - dont chacun sait aujourd'hui qu'il constitue une composante de notre nation. Elle s'interroge sur une agglomération, à tous les sens du terme, où l'islam joue un rôle important, intriqué avec d'autres enjeux - la Ville, l'éducation, l'emploi, la sécurité, la politique, la religion - pour former la notion même de « banlieue » dans l'usage courant du français contemporain. Nous avons choisi pour terrain d'enquête deux municipalités voisines dans le département banlieusard par excellence de Seine-Saint-Denis, Clichysous-Bois et Montfermeil, l'une composée en majorité de "grands ensembles ", l'autre de pavillons, assez différentes mais liées, justement, par une « communauté d'agglomération ».

Les banlieues de l'islam parcouraient en surface la forêt primaire de l'islam en France au milieu des années 1980 sans autre ambition qu'y tracer les layons pour se repérer. Banlieue de la République pousse sa tarière à travers les couches inextricablement imbriquées où se déploie l'islam de France un quart de siècle plus tard : l'habitat, en cités dégradées ou rénovées et en pavillons ; l'école, le collège et le lycée ; le travail et le chômage ; la tranquillité publique et l'émeute ; les réseaux associatifs, les élections municipales et cantonales ; la construction des mosquées, le Ramadan, le halal. Pour procéder à ce carottage en profondeur, il fallait se concentrer sur un territoire limité, dans lequel saisir l'épaisseur du lien social depuis sa dimension intime, prêter l'oreille, rendre la parole à des individus, dont la plupart sont citoyens français, mais dont la voix porte peu, étouffée par le discours que tiennent sur « les banlieues » ceux qui se prévalent d'autorité, sauf lorsque l'émeute en libère le cri - comme ce fut le cas en automne 2005, enflammant au départ de Clichy la France des quartiers sensibles. Notre pari, ici, est de contribuer à rendre ces quartiers intelligibles, en observant au quotidien comment s'y réalise - ou non - la promesse républicaine. De ce point de vue, nous posons en postulat de départ que Clichy-Montfermeil, c'est la France même. La banlieue n'est pas à la marge, mais au centre : c'est au prix de ce renversement de perspective que se lit notre avenir commun.

Il ne s'agit pas, bien évidemment, de projeter mécaniquement sur le macrocosme français les leçons que nous enseigne le microcosme de Clichy-Montfermeil. Cette agglomération n'est pas " représentative »- comme on le dit d'un sondage réalisé selon la méthode des quotas en administrant un bref questionnaire ; elle est emblématique, et nous l'avons pénétrée au fil des jours et des saisons d'une année, en passant de longues heures avec cent personnes de tous âges, origines et conditions, habitant ou ayant habité l'agglomération et ses environs. Les entretiens qu'elles nous ont accordés, dont la transcription couvre plus de deux mille pages, se sont déroulés en français pour la plupart mais aussi en arabe, en turc, en cambodgien, en anglais, en peulh, en soninké - la langue de l'ethnie du fleuve Sénégal la plus présente dans l'agglomération - au choix de l'interlocuteur. Deux tiers des 
enquêtés se disaient de confession musulmane, un tiers de confession chrétienne, bouddhiste, juive, ou sans religion1. Nous y sommes retournés pour les fêtes, les élections, les événements divers, et de multiples rencontres et visites de terrain, rusant avec les transports en commun et leurs multiples ruptures de charge pour parvenir vers cette banlieue enclavée et en revenir - à rebours des efforts quotidiens que doivent faire ses habitants pour en sortir afin d'accéder aux bassins d'emploi.

Clichy-Montfermeil a connu une notoriété universelle lors des événements de l'automne 2005, dont la survenue et surtout la propagation dans la France entière, en dépit des reportages des journalistes du monde et des nombreux essais qu'elles ont suscités, gardaient une part de mystère. Or ces émeutes, outre leur dimension spectaculaire, heurtaient en son tréfonds le Grand Récit fondateur de la France moderne, l'imaginaire implicitement partagé selon lequel la nation était toujours capable d'intégrer, quels que soient les aléas sociaux, culturels, ethniques, tous ceux qui étaient venus y résider et, plus encore, leurs enfants, nés sur le sol de leur nouvelle patrie, éduqués à l'école de la République, et donc imbus des valeurs communes qu'elle leur avait inculquées. Les émeutes, auxquelles le gouvernement répondit en décrétant l'état d'urgence, qui n'avait plus été utilisé sur le territoire métropolitain depuis cet autre moment de crise majeure de l'identité nationale que fut la Guerre d'Algérie, donnèrent le sentiment que le modèle d'intégration français ne fonctionnait plus, qu'il était enrayé. Avait-il perdu ses ressorts internes, touché par les effets dévastateurs d'une profonde crise économique ? Ou s'avérait-il impuissant face à des défis inédits - l'ampleur ou plutôt la nature des flux migratoires, et le rôle que jouaient en leur sein les modes d'affirmation de l'islam - quelques mois à peine après les attentats terroristes perpétrés par Al Qaïda à Londres, en juillet 2005, et au lendemain de la publication par un quotidien danois des caricatures du Prophète Mohammed, qui devaient susciter une intense mobilisation des musulmans à travers le monde ?

Les médias anglo-saxons eurent tôt fait de nommer les événements de l'automne 2005 Muslim Riots, en exacerbant la composante islamique à laquelle ils conféraient la dimension d'un universel conquérant, opposable à l'universalisme failli des Lumières dans sa version française et républicaine. A cette caricature, on opposa une première salve de travaux qui soulignaient la volonté des jeunes qui brûlaient les voitures pour attirer l'attention sur leur sort de crier leur appartenance à la société française dont ils avaient le sentiment qu'elle les rejetait. Et on montra, sondages à l'appui, en contrastant la propension des musulmans de France au mariage en dehors de la communauté, leur bonne opinion des juifs, etc. - par rapport à leurs coreligionnaires du Royaume-Uni ou du reste du monde - que les valeurs de la République étaient acceptées et l'intégration en marche.

L'enquête à Clichy-Montfermeil amène à remettre à plat ces deux postulats. On a pu en effet y observer les logiques d'une forte construction communautaire autour de la référence à l'islam au sein de laquelle s'expriment aussi bien des tendances centrifuges par rapport à la société française et à ses valeurs que des mouvements centripètes, mais qui sont fréquemment contrariés par l'adversité sociale. Entre ces deux pôles opposés, une vaste gamme d'attitudes de personnes qui cherchent à négocier au mieux leur situation, en fonction des ressources culturelles et matérielles dont elles disposent, dont certaines formulent des exigences identitaires dans le langage du halal, tentent d'adjuger leur vote en fonction du respect d'un agenda islamique par les candidats aux élections - mais aussi d'autres qui animent des mobilisations citoyennes avec une perspective laïque, s'engagent dans l'action associative pour favoriser l'accès à l'emploi, l'amélioration du cadre de vie dans les cités dégradées, l'éducation des enfants et la formation des adultes.

Clichy-Montfermeil est emblématique car s'y manifeste une collection de symptômes sociaux que l'on ne retrouvera ailleurs que latents et erratiques. C'est ce qui explique que les émeutes, par-delà l'événement conjoncturel - l'électrocution tragique de deux adolescents qui fuyaient la police - y aient trouvé les circonstances propices à déclencher l'embrasement généralisé, et c'est ce qui en rend l'observation particulièrement pertinente : l'agglomération permet de construire un « tableau de pensée », d'approcher un «type idéal » dans le vocabulaire de Max Weber, auquel mesurer ensuite les variations par rapport à d'autres banlieues et quartiers sensibles, ou par rapport à l'expression de l'islam en France en général. Ainsi par exemple, on verra qu'une très forte proportion de nos interlocuteurs musulmans se sont dits opposés au mariage avec un conjoint non-musulman - ce qui 
contredit tous les sondages pour lesquels la majorité de ces personnes, en France, accueille favorablement le mariage mixte -, ou que tous les hommes musulmans de notre échantillon à quelques exceptions près ont déclaré se rendre régulièrement à la mosquée - alors que la proportion sur la France entière ne dépasse guère le tiers de la population concernée (elle augmente toutefois régulièrement). Qu'est-ce qui explique pareils écarts, que nous disent-ils ? Lorsqu'on remarque qu'à Clichy une large majorité de la population est de confession musulmane, que la commune est enclavée par une médiocre desserte des transports en commun, que le chômage y bat des records, et que l'influence de mouvements et prédicateurs rigoristes s'y déploie depuis les années 1980, on dispose d'éléments de perspective et de mesure, qui peuvent nourrir la réflexion et le débat.

Outre la notoriété que vaut à l'agglomération d'être le lieu d'où sont parties les plus grandes émeutes de l'Histoire de la France contemporaine, celle-ci présente d'autres caractéristiques fortement contrastées. Sur le plateau que se partagent les deux municipalités et où ont été édifiés dès les années 1960 des grands ensembles au devenir calamiteux, des copropriétés dégradées où sévissent les marchands de sommeil qui logent dans l'illégalité des primo-migrants toujours plus paupérisés, a été réalisé le plus ambitieux des Programmes de Rénovation Urbaine de la nation - doté de plus de 600 millions d'euros - que le ministre de la Ville Jean-Louis Borloo qualifiait de " plat de résistance » de sa politique. L'investissement de l'État, des collectivités locales et des organismes de logement s'est traduit par une transformation complète du paysage, que nous avons pu observer entre l'été 2010 et l'été 2011, en voyant disparaître des tours et barres taguées aux ascenseurs cassés, entourées d'épaves de voitures, et surgir des résidences d'aspect agréable et bien tenues. Les habitants que nous avons interrogés à ce propos sont pour la plupart satisfaits de cette évolution, qui contribue à faire retrouver l'estime de soi, mais demeurent préoccupés par la perspective d'une nouvelle dégradation des logements, si l'éducation des enfants reste déficiente, si le chômage de masse persiste, si les politiques publiques ne se réorientent pas du béton vers l'humain. On a ici, à partir d'un cas exemplaire, la position d'un problème qui vaut pour l'ensemble de la réflexion sur le devenir de la Rénovation Urbaine - au moment où le premier plan s'achève en 2012 et où il faut définir les priorités du second.

En plein coeur du secteur de la Rénovation Urbaine avait été réservé un terrain pour édifier la Grande Mosquée de Clichy-Montfermeil, au terme d'un long processus de concertation entre les édiles et une fédération d'associations musulmanes pour faire émerger un islam consensuel dans un lieu de culte digne : il s'est arrêté au terrassement, tandis que les associations sont en procès entre elles et contre l'entrepreneur. Par-delà la fragmentation conflictuelle de l'islam de France, l'impécuniosité de la majorité de ses fidèles, l'échec de la Grande Mosquée est emblématique, là encore, des difficultés qu'engendre l'application à la lettre de la loi de 1905 qui interdit les subventions à la construction de lieux de culte mais autorise de financer les réparations de ceux qui furent édifiés antérieurement. Autre matière à débat : en effet l'agglomération compte une dizaine de mosquées improvisées dans des locaux en rez-de-chaussée de barres d'habitation, dans un entrepôt, une arrière-boutique, des pavillons réaffectés, mais la question de la prise en compte des équipements cultuels en complément de la Rénovation Urbaine - lorsque les populations concernées en expriment le besoin - se pose, et concerne en particulier, mais non exclusivement, les religions apparues le plus récemment, comme c'est aussi le cas pour les temples évangéliques.

L'inquiétude de nos interlocuteurs sur l'éducation à la civilité des enfants et des jeunes - perçue comme la condition nécessaire pour éviter que s'enclenche de nouveau le cercle vicieux des dégradations urbaines, ruinant la logique et obérant les investissements de la Rénovation - pose des problèmes fondamentaux sur l'éducation en banlieue difficile. Nous avons rencontré des parcours de réussite remarquables, grâce à l'aide et au discernement d'enseignants motivés, et aussi par la " débrouille » et la " niaque »; mais la situation de la majorité est préoccupante, et cela dès la petite enfance. Dans une agglomération où la fécondité des jeunes couples originaires du Maghreb s'est alignée sur celle de la population majoritaire en France, on demeure frappé par le taux de natalité élevé de familles primo-arrivantes venues de l'Afrique sahélienne, dont les mères travaillent dur de longues heures, et dont les enfants en bas âge sont sous-encadrés par le système éducatif, mettant d'emblée en péril leur insertion sociale à court terme. Là encore, l'équipement en crèches et écoles maternelles pour ces populations, dont les classes ont été supprimées en nombre ces dernières années, est un débat public central pour notre société, par-delà les quartiers concernés. Plus loin dans le parcours éducatif, 
la question de l'orientation reste perçue comme traumatique par nombre de nos interlocuteurs - voués à des filières professionnelles, sans avenir selon eux, par un mode de sélection dont ils incriminent le caractère ségrégatif. Cette mise à distance des établissements d'enseignement, leur altérité, ont été renforcées par le délitement de ces passerelles qu'assurait l'encadrement para-scolaire autrefois pourvu par le mouvement ouvrier, contribuant aujourd'hui à l'isolement de l'école dans le tissu social. La sous-fréquentation de la demi-pension en est un exemple, lorsqu'elle se cristallise sur le respect du halal et, à ce motif laisse trainer les jeunes nourris d'un sandwich aux normes religieuses dans les coursives des cités. Un autre est la question du port du hijab : si la loi issue des recommandations de la «Commission Stasi » a été acceptée, elle n'a pas été comprise, et cela d'autant que les propositions d'accompagnement - du respect de fêtes religieuses non chrétiennes aux enseignements des langues d'origine - sont restées lettre morte, alors qu'elles participaient de la dimension égalitaire de la laïcité républicaine et de l'insertion de notre société dans un monde globalisé.

Le coeur du problème de Clichy-Montfermeil est l'emploi - comme pour l'ensemble de la société française, mais l'agglomération en représente un cas d'espèce, exacerbé par l'enclavement, la difficulté des transports qui éloigne les lieux de travail, et le décalage entre les compétences que requièrent certaines zones actives et prospères, qui se déploient en Seine-Saint-Denis sur les friches de la désindustrialisation, et le capital éducatif et culturel inadapté d'une grande partie de la jeunesse qui n'y est pas «employable ». L'enquête marque le contraste entre des itinéraires de réussite dont certains s'appuient sur la performance scolaire, d'autres sur la mobilisation de réseaux - comme c'est le cas pour les entrepreneurs turcs - d'une part, et des parcours de galère, d'autre part. Parmi ces derniers, si les cas les plus lourds sont représentés par ceux qu'éjecte le système scolaire au sortir du collège, les exemples les plus préoccupants pour le devenir de la société sont incarnés par les diplômés de l'enseignement du supérieur qui, parfois mal orientés, toujours dépourvus de réseaux de connaissances, quelquefois victimes de discriminations qui vont du faciès au code postal, ne parviennent pas à trouver du travail correspondant à leurs compétences, en viennent à retourner le stigmate dont ils souffrent en un rejet radical de la France et des valeurs qui lui sont prêtées. Qu'ils désirent quitter l'Hexagone pour de bon ou y propager la révolte, nous avons plusieurs fois rencontré, au substrat de ces logiques de rupture, l'affirmation de valeurs islamiques de clôture communautaire, de protection face à l'adultération délétère reprochée à la société.

C'est l'alchimie complexe de ces ruptures, des frustrations, des demandes d'intégration sociale, que l'on retrouve dans les émeutes de l'automne 2005 et dans les comportements de déviance qui vont des incivilités à une économie parallèle dont le trafic de stupéfiants est l'aspect le plus saillant. Il emplit le propos de nos interlocuteurs les plus désemparés qui ne peuvent plus rentrer chez eux sans se faire importuner par les dealers qui squattent les halls d'immeuble, comme la malédiction de trop d'un destin adverse, motif à déplorer l'impuissance d'une police controversée. Très critiquée parfois pour ses opérations " coup de poing 》 et son enfermement dans une culture institutionnelle en déphasage avec la population, mise en cause à l'occasion du déclenchement des émeutes, elle fait paradoxalement l'objet d'une forte demande de beaucoup d'habitants, qui ont plébiscité l'ouverture d'un commissariat à Clichy à l'automne 2010 comme la marque que prévention et dissuasion contribueraient significativement à une tranquillité publique à laquelle ils aspirent en masse - au contraire d'une répression décrite comme indiscriminée voire aveugle. C'est là du reste que se noue le double point de départ des émeutes. Si chacun a en tête l'électrocution des deux adolescents, le 27 octobre 2005, qui suscita la révolte de leurs pairs, rapidement calmée mais point éteinte par l'intervention des grands frères et des adultes, c'est un événement moins connu, le "gazage de la mosquée Bilal » trois jours plus tard, qui relança le feu couvant sous la cendre et embrasa le pays. Une grenade lacrymogène tirée par la police à proximité de cette mosquée située dans un ancien entrepôt et qui asphyxia les centaines de pieux adultes massés là pour la prière nocturne du Ramadan suscita l'indignation généralisée, faisant basculer la génération des « darons » [parents] du côté des jeunes, en commençant par les villes voisines du 93 peuplées de populations de mêmes origines locales, puis se propageant dans tous les « quartiers sensibles ». Nos interlocuteurs les plus engagés ont construit en rétrospective un Grand Récit des émeutes, chargé d'hyperboles où le « gazage » évoquait la répression des Palestiniens par Israël à Gaza, voire le gazage des juifs dans les camps nazis - par une réappropriation mimétique de la persécution antisémite dont nous verrons les effets politiques -, traduisant le mépris de l'Etat pour les 
musulmans à qui il avait déclaré une manière de guerre ravivant les souvenirs de l'Algérie. Ils nous ont aussi décrit comment le temps du Ramadan rythmait les échauffourées, le caillassage des CRS commençant comme un rituel de lapidation chaque soir après le repas d'iftar rompant le jeûne diurne, pendant que les parents partaient prier à la mosquée. Mais si le vocabulaire de l'émeute empruntait au registre sémantique de l'islam, la finalité n'avait rien à voir avec Ben Laden contrairement aux élucubrations entendues ici ou là. Le ressort en était social, tenait, comme nous le verrons, au déni d'intégration par la société globale, notamment dans l'accès au travail - et tous les participants aux émeutes n'étaient pas musulmans ; mais la solidarité communautaire constituait l'une des ressources de la mobilisation, d'autant que le déclencheur en avait été l'agression contre une mosquée, magnifiée en rétrospective pour sacraliser et légitimer la révolte. Nous avons entendu aussi la parole de ceux qui ont subi les émeutes : les groupes minoritaires, turcs, pakistanais, chrétiens d'Orient, dont certains individus étaient terrifiés par la violence des jeunes d'origine maghrébine ou africaine qui ravivait la mémoire d'autres conflits ethniques locaux liés à la prédation, ou à des pogroms au pays, cherchant à éviter l'incendie de leur voiture en accrochant un Coran au rétroviseur ou en étalant un tapis de prière sur la plage arrière. Selon d'autres, l'ampleur circonscrite dans le temps et l'espace de l'émeute avait fait l'objet d'un traitement disproportionné et pervers dans les médias, piégeant ceux-là mêmes qui y voyaient le vecteur d'une prise de parole publique des sans-voix, et, en terrorisant par les images de voitures brûlées l'électorat de la France profonde comme des beaux quartiers, assurèrent in fine l'élection à la présidentielle de 2007 de celui-là même qui était ministre de l'Intérieur durant cet épisode.

Au lendemain des violences, Clichy-Montfermeil fut à l'avant-garde d'un flux massif d'inscription des jeunes Français issus de l'immigration sur les listes électorales, notamment grâce à un mouvement de mobilisation civique dont l'éloquent acronyme d'ACLEFEU [assez le feu !], pour « Action Collectif Liberté Égalité Fraternité Ensemble Unis », disait assez bien la volonté de traduire des émeutes qu'il avait rebaptisées « révoltes sociales » en agir politique. S'inscrivant dans la filiation de la Marche pour l'Égalité et contre le Racisme (connue par son surnom de « marche des Beurs ») et autres actions des années 1980, organisant cahiers de doléances et collectes de signatures à travers la France, le Collectif appela à voter pour Ségolène Royal - qui était venue à Clichy signer sa charte - au second tour de l'élection présidentielle de 2007. Si celle-ci y recueillit plus de $60 \%$ des voix tandis que le taux de participation local record culminait à $82 \%$, son échec national se traduisit dès les législatives suivantes par une forte démobilisation - dans une circonscription découpée en 1986 pour sécuriser l'élection d'un député de droite en accompagnement de la déprise du communisme. Si ces inscriptions massives rééquilibrèrent quelque peu pays légal et pays réel, en dépit de la masse des mineurs (dont ceux d'origine immigrée sont nombreux) et des étrangers réguliers ou clandestins, elles firent des citoyens et électeurs de confession musulmane, pour autant qu'ils votaient, un enjeu et une ressource, une composante de l'électorat, au même titre que les électeurs du Front National, ou ceux de confession israélite ou chrétienne. Et la construction des mosquées - auxquelles s'opposait le parti de Marine Le Pen - devint une thématique que les programmes et professions de foi de ses adversaires devraient peser au trébuchet avec les questions de l'immigration ou de l'insécurité - rendant plus complexes les déterminants du vote.

Nous avons observé comment la «course à l'échalote » des candidats à l'élection cantonale de mars 2011 dans l'agglomération se déroulait aussi bien lors de l'Aïd el Kébir que pour Yom Kippour, et dans une moindre mesure lors du pèlerinage catholique annuel clichois de Notre-Dame-des-Anges. Mais aussi comment la reprise par un candidat du vocabulaire de l'extrême droite lui avait aliéné les sympathies des jeunes « issus de la diversité », dont certaines lui étaient pourtant acquises, contribuant à son échec, tandis que, dans un autre canton, le candidat écologiste avait rehaussé le vert de ses suffrages par celui des voix qu'orientait en chaire le prédicateur d'une mosquée indiquant le « bon choix » à ses ouailles.

Ce passage au politique en cours de mutation profonde qui voit une nouvelle population française commencer de se faire entendre dans les urnes s'accompagne d'interrogations en profondeur sur l'identité nationale : notre enquête a eu lieu peu après un débat controversé sur ce thème, initié par un ministère qui porta cet intitulé pour disparaître en novembre 2010, et au lendemain des déclarations du président de la République à Grenoble en juillet de cette année proposant de déchoir de la nationalité 
française certaines catégories de criminels naturalisés. Nombre de nos interlocuteurs - y compris ceux qui, d'origine étrangère, jouaient le jeu de l'assimilation - se sont sentis stigmatisés par la façon dont ces thématiques étaient utilisées pour " gagner les élections sur notre dos »; d'autres ont réitéré avec force leur identité française, et certains n'ont vu leur salut que dans un départ définitif de la France dès que l'occasion s'en présenterait.

Si l'islam, confession majoritaire à Clichy, est un enjeu majeur dans l'agglomération, il n'y est pas seul : le maire de Montfermeil, membre du Parti Démocrate-Chrétien et confortablement élu, affiche des valeurs catholiques au nom desquelles il s'est prononcé plusieurs fois contre la dimension conquérante de l'islam du jihad - et a décrit en termes de combat de cultures des émeutes advenues sur sa commune et des agressions le visant directement. Il n'en a pas moins, trois mois plus tard, signé la promesse de vente du terrain destiné à la Grande Mosquée - afin que les musulmans disposent d'un lieu de culte digne et adapté. Le moindre des paradoxes n'est pas du reste que cette communauté d'agglomération fonctionne très harmonieusement alors que les édiles des deux communes sont de bords politiques opposés - même si l'appartenance de Clichy et de Montfermeil à deux cantons différents réduit les frictions électorales. En ce sens aussi, notre microcosme cristallise voire pousse à la limite, sur son petit territoire, les interactions qui se produisent dans la société au sens large.

Par rapport à l'enquête des Banlieues de l'islam de 1985, si l'une des transformations majeures en un quart de siècle est la citoyenneté française de la majorité des personnes de confession musulmane résidant dans l'Hexagone, accompagnant le passage à l'âge adulte d'une génération qui n'était alors pas encore née ou trop jeune, l'autre est l'ubiquité du halal dans cette population. Une fois encore, les traits en sont plus accentués à Clichy qu'ailleurs, mais de peu, tant cette revendication identitaire a explosé. Le halal, comme on le verra, a un spectre beaucoup plus vaste que la viande et touche aussi, plus profondément, à la chair ; il fait passer de la table au lit, et construit des repères passablement complexes pour définir le licite et l'illicite. Ses frontières se réclament d'autant mieux d'un absolu et d'interdits sacro-saints qu'elles sont mouvantes dans la réalité, faites d'accommodements raisonnables ou non, très corrélés aux situations sociales, et dans l'agglomération, extrêmement liées à la situation d'enclavement, au contrôle social et à l'ordre moral quotidien qui en découlent - auquel du reste les jeunes couples s'empressent d'échapper en s'installant dans l'anonymat des communes limitrophes.

Si le halal constitue un fort marqueur communautaire, c'est aussi qu'il s'est construit, dans la France du $21 \mathrm{e}$ siècle, en miroir inversé du casher. Tout au long de l'enquête, les juifs apparaissent, chez beaucoup de nos interlocuteurs musulmans, comme une minorité qui a su imposer sa spécificité - d'où elle tirerait la puissance qui lui est prêtée en dépit de son faible nombre, la crainte qu'elle inspire, le respect qui lui est prodigué. Que l'on oppose le port de la kippa à celui du voile à l'école, l'émotion que suscitent la profanation des synagogues ou le meurtre d'un juif et l'indifférence au " gazage de la mosquée » ou à l'assassinat d'un musulman en 2010, il existe une sorte de compétition mimétique qui s'accompagne de remarques sur le peu de considération fait aux chrétiens par la société. Ceux-ci ne sont «plus très croyants » déplorent certains de nos interlocuteurs musulmans, qui ne sauraient donc se comparer à eux sauf à accepter le destin d'une dilution identitaire dans une laïcité dont les références éthiques, la dimension d'égalité ne sont plus perçues clairement par des populations qui vivent au quotidien les inégalités sociales.

Sans doute est-ce la faible capacité d'attraction de la promesse laïque qui interroge le plus au terme de cette recherche : mais la laïcité a-t-elle un corps qui ne soit pas social - comme le dit son beau nom grec, laïkos, qui signifie qu'elle est « du peuple » ? Il faudrait pour cela que l'insertion dans la société par l'emploi rende au peuple dans sa diversité une pleine croyance dans les valeurs de la nation, et que l'éducation lui en ait fourni les capacités, face aux défis quotidiens d'un univers mondialisé et postindustriel qui bouleverse les repères d'hier. C'est le chantier immense qui, après la Rénovation Urbaine, s'ouvre dans les quartiers défavorisés : l'enjeu en est l'homme, dont seul le travail fait un citoyen à part entière, et cela se ressent avec une acuité emblématique dans la banlieue qui a accueilli notre enquête, à Clichy-Montfermeil, au coeur de notre République. 


\section{Chapitre 1 : La Rénovation Urbaine}

La Rénovation Urbaine constitue aujourd'hui le principal moyen d'action financier à destination des banlieues dégradées. Initié en 2004, le Plan de Rénovation Urbaine (PRU) de la communauté d'agglomération de Clichy-sous-Bois et de Montfermeil, d'un montant de près de 600 millions d'euros, est le plus important PRU de France. Si ce plan s'inscrit dans la continuité d'une politique de réhabilitation lancée depuis les années 1980, il reste perçu par de nombreux des habitants de l'agglomération comme une réponse immédiate et nécessaire aux émeutes de 2005. Dans ses principes, il entend restaurer l'estime de soi des habitants en leur assurant des conditions d'habitation plus dignes, premier pas vers une meilleure intégration sociale, et posant les bases du sentiment d'appartenance à la République.

\section{Un cadre de vie transformé en profondeur}

Fondé sur un ensemble de démolitions, de réhabilitations, de reconstructions de logements et de restructuration de la voirie, la forte visibilité et la dimension spectaculaire de ces transformations sont essentiellement perçues de manière positive par les habitants. La plupart reconnaissent un saut qualitatif lié à la réhabilitation de la Zone Urbaine Sensible du «Plateau », regroupant 15000 résidents. Le processus de «résidentialisation » qui se traduit par une rénovation des appartements existants et des parties communes, ainsi que par une meilleure délimitation des espaces privé et public (installations de barrières, grilles, digicodes) a permis un gain de tranquillité et une sécurisation des espaces intermédiaires.

L'ouverture d'un commissariat à l'automne 2010 constitue également une étape majeure dans ce processus de sécurisation. Souhaïla, lycéenne de 17 ans, approuve sans réserve le changement du paysage urbain : "Je trouve que c'est une ville en rénovation, c'est une ville en mouvement. Ca me fait vraiment plaisir. (...)Y a des infrastructures juste à côté de mon lycée donc je le vois de jour en jour. Un commissariat qui a poussé comme un champignon. Regardez tous ces bâtiments-là. Et jusque le MacDo qui a été rénové aussi !».

\section{La Rénovation Urbaine doit faire face à une réalité sociale difficile}

La communauté de Clichy-Montfermeil compte officiellement 60000 habitants, cependant ce nombre reste approximatif du fait des sans-papiers. La population de l'agglomération se caractérise globalement par sa pauvreté, par une proportion importante d'immigrés et de leurs descendants et par le nombre très significatif de familles de grande taille.

La structuration de l'habitat diffère sensiblement dans les deux villes et cet écart est accentué par les différentes stratégies initiées par les forces politiques locales. Montfermeil est aux trois-quarts pavillonnaire et sa population dispose d'un revenu imposable par ménage deux fois supérieur à celle de Clichy-sous-Bois. Cette dernière est constituée aux trois-quarts de cités et détient le record du plus grand nombre de mineurs ayant au moins un parent né à l'étranger ( $76 \%$ - contre $50 \%$ à Montfermeil et $57 \%$ en moyenne en Seine-Saint-Denis).

Depuis plusieurs décennies, Clichy-sous-Bois a vu le départ des natifs et des immigrés d'origine européenne et maghrébine vers les communes limitrophes, compensée par l'arrivée via le regroupement familial de familles essentiellement originaires d'Afrique subsaharienne. Ce processus contraint Clichy-sous-Bois à une très faible mixité sociale. La Mairie de Montfermeil quant à elle, a opté pour un processus de gentrification autour des zones pavillonnaires et pour une valorisation de son identité française et de son caractère chrétien. Les pavillons, qui rassemblaient à l'origine des habitants natifs ou originaires de l'immigration européenne (italienne et portugaise) accueillent de manière plus récente des familles d'origine maghrébine, turque ou levantine. 
Les habitants de Clichy-Montfermeil sont répartis dans quatre principaux types d'habitation : copropriétés dégradées, logements sociaux anciens, logements sociaux issus de la Rénovation Urbaine, pavillons et propriétés bien entretenues. La majorité de l'habitat concerné par le PRU est constitué de copropriétés dégradées, comme l'illustre le cas de la copropriété de la Forestière à Clichy-sous-Bois. Construit en 1975, plutôt à destination de cadres, sur la base d'un projet de voie rapide qui n'a jamais vu le jour, cet ensemble immobilier a subi une «spirale de la dégradation » : le défaut d'entretien a provoqué une baisse des prix de l'immobilier, le départ des propriétaires solvables et l'arrivée de bailleurs peu scrupuleux. Du fait de sa proximité avec l'aéroport de Roissy-Charles de Gaulle, la copropriété est devenue la proie des marchands de sommeil et un lieu de concentration de primoarrivants.

Parallèlement, la dégradation des biens s'est accompagnée d'une hausse du prix de l'énergie, entraînant un surendettement des propriétaires. Ce processus, observé ailleurs également, interroge le sens d'une politique d'accession à la propriété des couches les plus fragiles de la population, si elles n'ont pas été sensibilisées à leurs responsabilités, et notamment au poids des charges. La plupart des grands ensembles de Clichy-Montfermeil, construits à la va-vite après la Seconde Guerre mondiale dans un contexte de forte spéculation immobilière, ont dans leur grande majorité connu cette spirale de la dévaluation. Dans ce contexte, l'objectif du PRU revient donc à accompagner des copropriétaires surendettés dans leur transition vers un habitat social rénové. Cette transition est parfois vécue comme une régression - du statut de propriétaire au statut de locataire - et peut s'avérer traumatique dans certains cas de relogements hors de Clichy-Montfermeil.

\section{Espoirs et frustrations}

Loin de l'image catastrophiste que l'on se fait d'ordinaire des banlieues populaires et que véhiculent à l'envi les médias, la plupart des habitants considèrent que lorsque le cadre quotidien est transformé par la Rénovation Urbaine, les conditions d'existence ne sont pas si désagréables. Une grande partie des habitants interviewés restent attachés à leur ville, aux modes de convivialité et aux réseaux humains qui s'y sont développés ainsi que le souligne Ismaïl, étudiant et assistant d'éducation: "Moi, je souhaite rester au quartier car... car je suis attaché au quartier. J'y ai toute ma jeunesse. J'ai grandi dans ce quartier. [...] J'ai envie de m'investir dans ce quartier. Quand je vois les jeunes, c'est comme si c'est tous mes petits frères, quoi. J'ai envie de m'investir dans la ville de Clichy-Montfermeil! »

L'enquête met toutefois en lumière trois axes d'incertitudes majeurs. D'une part, la nature des relogements proposés n'est pas sans provoquer des déceptions liées au sentiment d'inadéquation de l'habitat rénové avec les attentes logistiques et financières des résidents. D'autre part, la rénovation ne touche pas l'ensemble des deux villes, mais seulement la partie commune à Clichy et Montfermeil que l'on appelle « le Plateau ». Pour Ibrahim, copropriétaire du Chêne Pointu dans le Bas-Clichy - un grand ensemble qui n'a pas été inclus dans le PRU -, retraité du BTP et de nationalité marocaine : «la rénovation, c'est passé ailleurs. Ici, on n'a jamais de rénovation [...]. Pour nous, il n'y a rien de fait à ce jour. Les gens, ils reçoivent la flotte sur la tête, les champignons dans les appartements, la propreté, les ascenseurs qui ne fonctionnent pas. Les gens sont là mais ne savent pas où aller. Ils n'ont pas les moyens de partir.»

Plus fondamentalement, nombreux sont ceux qui craignent que la spirale de la dégradation constatée depuis les années 1960 ne se reproduise dans les nouveaux habitats. Pour Hassan, originaire du Maroc, diplômé de l'enseignement supérieur et sans emploi : "Pour l'instant, ça change le paysage. C'est tout ce que ça change pour l'instant. Faut pas oublier qu'on a pris les mêmes, et qu'on a recommencé, les mêmes habitants... ». La crainte reste vive que le PRU ne soit pas articulé à des dispositifs permettant l'éradication des maux sociaux structurels ayant conduit à la dégradation des conditions de vie dans les anciennes cités HLM et les copropriétés. C'est la capacité de la Rénovation Urbaine à s'articuler avec le social et à agir sur les maux structurels que sont le chômage, l'enclavement et l'absence de mixité sociale qui garantira sa réussite comme l'exprime Abbas, responsable associatif : "Ça change le paysage de la ville. C'est sûr que ça change. On est contents de voir ces beaux 
immeubles sortir de terre. Et puis une architecture nouvelle plus moderne, ça coupe. C'est une nouvelle page qui s'écrit. On n'est plus dans les barres des années 60. Voilà. Ça, c'est la forme. Mais, sur le fond, on s'attaque pas aux réelles causes. C'est pas ça qui va changer nos vies dans les quartiers. C'est pas ça qui... Tant qu'on ramènera pas le transport, le travail, les services publics, le droit public, on n'aura pas réglé le problème. » 


\section{Chapitre 2 : L'éducation}

L'éducation constitue un enjeu majeur de la réussite dans les quartiers populaires. Elle seule permettra d'assurer l'adéquation entre les exigences toujours plus grandes d'un univers où la concurrence pour les compétences est mondialisée et où l'offre d'emplois est poussée vers le haut. Face à ces défis, la jeunesse de Clichy-Montfermeil, d'une bonne partie de la Seine-Saint-Denis et des zones urbaines en difficulté, dispose potentiellement d'atouts considérables pour la France de demain, ne serait-ce que sa dynamique démographique, mais ses capacités restent globalement déficientes en raison d'un niveau culturel trop faible. Ce potentiel n'est pas réalisé comme il le pourrait, alors que des solutions peuvent se dégager rapidement, notamment pour une meilleure articulation de l'éducation avec les autres dispositifs de la Politique de la Ville - au moment où la Rénovation Urbaine doit s'engager dans l'investissement humain pour éviter la dégradation de ses réalisations.

\section{Echec scolaire et milieux défavorisés : le problème français}

Depuis trente ans, les politiques d'éducation prioritaire mises en œuvre pour porter remède aux difficultés de l'école à transmettre efficacement les savoirs dans les quartiers défavorisés sont soumises à de sévères critiques. Elles donnent le sentiment d'avoir été menées, pour une large part, sans véritable lien avec la Rénovation Urbaine. La situation des élèves en plus grande difficulté ne s'est pas améliorée et la France, en plus d'être le pays de l'OCDE où le retard scolaire à quinze ans est le plus important, est celui où l'impact de l'origine sociale sur les élèves est le plus élevé. $20 \%$ d'une classe d'âge quitte le système scolaire sans diplôme (environ 150000 personnes par an). Ces décrocheurs risquent d'entrer dans un cycle d'exclusion sociale qui conditionnera leur vie et celle de leurs enfants.

Clichy-Montfermeil a fait partie du premier groupe de territoires classés en Zone d'Education Prioritaire (ZEP) dès 1981. Malgré les moyens affectés et les différents dispositifs mis en œuvre depuis trois décennies, le territoire scolaire de Clichy-Montfermeil reste très en-deçà des taux de réussite nationaux. Plus de la moitié des élèves de l'agglomération, au sortir du collège, sont orientés en CAP ou en BEP et ne parviennent à intégrer ni le lycée général, ni le lycée technologique. Les handicaps sociaux et culturels y sont particulièrement lourds et une ségrégation de fait sépare les «collèges pour pauvres" classés en ZEP à Clichy-sous-Bois (et de manière plus nuancée à Montfermeil où enfants de la cité des Bosquets et enfants des pavillons fréquentent les mêmes établissements) des « collèges pour riches » des communes voisines à prédominance pavillonnaire.

\section{Les aléas de l'orientation}

Apparaissant pour de nombreux enquêtés comme le seul levier de l'ascension sociale et porteuse d'espoirs immenses, l'école est également l'objet des ressentiments les plus profonds en cas d'échec scolaire, apparaissant comme le lieu de reproduction des inégalités sociales. La figure la plus détestée par nombre de jeunes est celle du conseiller d'orientation à la fin du collège, loin devant les policiers. Il cristallise sur sa personne l'inadéquation entre formation et insertion sociale. Ainsi en témoigne Hamza, informaticien d'origine turque : "Les trois-quarts de mes copains, ils ont été éjectés en $B E P$ ! 》 ou encore Marwan, 28 ans, d'origine marocaine, chauffeur de bus : "Le prof avait convoqué mon père [...]. Il lui a dit: "Je vois que votre fils, il a 14 en technologie [...]. C'est pas le top mais c'est pas mal [...]. Il devrait peut-être faire un BEP en électronique [...]". Mon père a vite réagi. Il a dit: "Attendez, il a 14 de moyenne et vous voulez l'envoyer vers une voie professionnelle!" Parce qu'une voie professionnelle, on voyait ça comme étant la débauche en fait. C'est vraiment: " tu vaux rien, alors tu vas en BEP". Alors que c'est pas nécessairement le cas parce même ceux qui arrivent en $B E P$ se dévalorisent, se voient comme étant des cancres alors que non. »

Face à ces échecs dans l'orientation, d'autres enquêtés témoignent au contraire de la capacité des enseignants à détecter les talents, à l'instar de Murat, 35 ans, d'origine turque, l'un des chefs 
d'entreprises les plus prospères de la zone franche urbaine: "A l'époque, on avait une très bonne orientation. On avait des profs qui étaient très présents et qui savaient aussi cerner, je dirais, en fonction de chaque personne, chaque lycéen. »

\section{La socialisation à l'école}

L'école ne se limite pas aux enseignements que l'on y reçoit. Elle constitue aussi un lieu de socialisation majeur avec le groupe des pairs et d'apprentissage par les élèves de leur future citoyenneté, des règles communes, des droits et devoirs au sein de la cité - au sens politique. Beaucoup de jeunes gens originaires des quartiers défavorisés, principalement d'origine maghrébine et turque, ont gardé un fort réseau de socialisation lié au quartier et remontant à l'école et au collège.

L'un des temps de l'articulation à l'école entre enseignement et socialisation est la demi-pension - la " cantine ». Conçue pour faciliter la tâche aux parents qui travaillent, elle fournit également un espace de encadré et surveillé. Or dans les cités de notre enquête, la restauration scolaire fait l'objet d'une très faible fréquentation et cristallise paradoxalement les questions sociales qu'elle avait vocation à faciliter. L'arbitrage économique en défaveur de la cantine scolaire - dont le coût revient souvent plus cher que le repas cuisiné à la maison par la mère au foyer - s'accompagne souvent d'un argumentaire culturel. En effet, certaines familles refusent la cantine, considérée comme n'offrant pas de nourriture halal. D'autres trouvent des accommodements raisonnables entre foi et vie sociale, à l'instar de Salima, lycéenne de seize ans d'origine marocaine : "Je suis dans un lycée public. Il faut demander si le repas est sans ou avec porc. Il faut être actif.» L'enjeu que représente la fréquentation de la demipension pour l'intégration culturelle et sociale, ainsi que pour l'adhésion des enfants et des familles au projet global de l'éducation est important.

L'un des débats les plus passionnés concernant la mise à distance culturelle de l'école et la relativisation des valeurs qu'elle porte a tourné autour du port du hijab par des jeunes filles musulmanes, depuis la première «affaire du voile » de l'automne 1989 au collège Gabriel Havez de Creil, dans l'Oise. Nourrie d'avis contradictoires du Conseil d'Etat, de prises de positions politiques multiples, la polémique a abouti à la nomination d'une commission de réflexion « sur l'application du principe de laïcité dans la République » par Jacques Chirac, alors Président de la République, au printemps 2003, dite «commission Stasi »-qui a formulé un certain nombre de recommandations. Seule celle qui concernait la prohibition des signes religieux ostentatoires dans l'espace scolaire a eu force de loi. Nombre d'enquêtés musulmans comme Hassan, d'origine marocaine ont le sentiment qu'elle ne vise que les musulmans : "Officiellement, elle était pour tout le monde. Mais, on sait très bien qui c'est qu'elle vise. J'ai travaillé dans le milieu scolaire. Je voyais même des profs avec des croix, c'est pas pour autant qu'on leur interdisait d'entrer. " L'un des points les plus importants qui ressortent de l'enquête est que la loi est respectée ; mais il est également notable que pour un grand nombre de répondants, la loi n'a pas été comprise, surtout chez les musulmans, mais pas exclusivement, et le déficit d'explication qui a suivi les délibérations de la "commission Stasi » est patent.

Cette commission avait pourtant proposé un certain nombre de mesures destinées à réaffirmer la dimension intégratrice de la laïcité. Or, en choisissant de ne mettre en œuvre que l'interdiction, sans suivre les autres recommandations, l'architecture d'ensemble a été déséquilibrée. C'est cela qui a rendu difficilement acceptable la loi chez un certain nombre de personnes qui, à l'instar de Hassan, trentenaire d'origine marocaine, estiment que «la laïcité n'est qu'un mythe. (...) On ne peut pas leur en vouloir, mais quand on parle de laïcité il faut le faire avec tout le monde. Faut pas me faire le Vendredi saint à la cantine. Faut pas caler toutes les vacances scolaires et les jours fériés sur les fêtes chrétiennes, par exemple ». L'adhésion aux valeurs de la laïcité comme mode inclusif du vivreensemble constitue un pré-requis culturel fondamental, sans lequel les conflits sociaux et les difficultés liées à l'échec scolaire, risquent de se réifier autour de clivages communautaires sur le territoire de la République. 


\section{Chapitre 3 : L'emploi}

S'il est un révélateur des difficultés rencontrées en France par la Politique de la Ville depuis 20 ans, c'est bien l'emploi. Sans insertion sociale par l'emploi, les opérations de rénovation urbaine n'ont au mieux que des effets transitoires sur des territoires en situation de forte relégation. Or, en 2009, les Zones Urbaines Sensibles (ZUS) ont enregistré un taux de chômage moyen de 18,6 \% contre 9,8 \% ailleurs en France ; chiffre qui monte à $43 \%$ pour les jeunes actifs ( $37 \%$ pour les jeunes actives). Outre les situations de pauvreté que ces chiffres induisent - près de la moitié des moins de 18 ans résidant en ZUS vivent sous le seuil de pauvreté, ils sont également vecteurs de phénomènes de crispation identitaire. L'absence d'emploi, la précarité et les "petits boulots» sans perspective constituent souvent un élément déterminant dans les conduites déviantes (délinquance, violence, trafic de drogues), le repli sur une identité religieuse fermée voire la revendication affirmée d'une rupture sociale et culturelle avec la société française.

\section{Un territoire cumulant les difficultés}

Les années 1970 marquent le tournant de la désindustrialisation dans les banlieues nord de Paris. La France a accéléré sa mutation vers l'économie tertiaire et la Seine-Saint-Denis voit ses grandes usines fermer les unes après les autres. Le déclin du Parti communiste et de ses structures d'encadrement social vont de pair avec la désindustrialisation. Entre le travail à l'usine et les emplois valorisés du secteur tertiaire, difficilement accessibles sans qualification, demeure aujourd'hui une "zone grise » pourvoyeuse de beaucoup de métiers faiblement rémunérés, pratiqués par nombre de personnes ayant répondu à notre enquête, faute de mieux : gardiennage, sécurité, ménage, services à la restauration, textile, BTP - tous «boulots» caractérisés par une forte précarité. L'aéroport Roissy-Charles de Gaulle, desservi directement par une ligne d'autobus depuis Clichy-Montfermeil, constitue le pôle principal d'activité pour ce type d'emplois, suivi de Paris, Marne-la-Vallée et la Plaine-Saint-Denis.

Territoire caractérisé par la déconnection spatiale entre lieu de résidence et lieu de travail, l'agglomération de Clichy-Montfermeil souffre en outre, au sein du département, d'un fort enclavement géographique qui l'empêche de bénéficier du dynamisme des pôles économiques existant en Seine-Saint-Denis. En outre, il n'existe pas d'agence de Pôle Emploi sur le territoire de ClichyMontfermeil - les agences les plus proches sont à Livry-Gargan et au Raincy. C'est la Communauté d'agglomération qui a créé la DEFI (Direction de l'Emploi, de la Formation et de l'Insertion), service public qui prend en charge notamment tous ceux qui ont renoncé à s'inscrire à Pôle Emploi.

\section{Des trajectoires d'insertion contrastées}

Notre enquête permet de distinguer et de mettre en valeur des trajectoires d'insertion par le travail fortement contrastées.

Des trajectoires de réussite tout d'abord. Elles sont le fruit d'une stratégie familiale délibérée d'investissement dans l'éducation, que l'on retrouve souvent dans les familles d'origine turque et maghrébine. Les personnes interrogées sur leur réussite mettent toutes en avant un soutien familial fort. Ainsi Ece, d'origine turque: "Dans les autres pays, quand on était une fille, on n'étudie pas beaucoup. Moi, j'ai eu la chance d'avoir une famille qui était civilisée et qui ne disait pas "toi tu es une fille, toi tu es un garçon" (...). Du coup mon père m'a dit: "Moi, je n'ai pas eu la possibilité d'étudier, alors vas-y. Etudie comme tu veux" ». Pierre, également d'origine turque : "Aujourd'hui, c'est mes parents que je remercie. Parce que c'est grâce à mes parents que j'ai réussi ». Elles sont aussi le fruit d'une détermination personnelle sans faille. Lila, jeune femme d'origine marocaine: "J'étais commerciale, marketing, télémarketing, baby-sitting, tous les boulots où j'ai pu épargner. Parce que j'avais mon objectif: aller aux Etats-Unis. (...) Au retour, j'avais eu un MBA, et après une grande banque américaine à Paris (...). Et après, j'ai fait un second cycle à Sciences Po en master 
Finance, dix mois, et après dans le Golfe ! ». La condition et la conséquence de cette réussite scolaire puis professionnelle sont souvent l'arrachement à son milieu, à son quartier.

La réussite passe également par le développement de l'entrepreneuriat. Ainsi les ZUS ont-elles été particulièrement réceptives au dispositif des auto-entrepreneurs mis en place par la loi de modernisation de l'économie d'août 2008. De même, sur le territoire de Clichy-Montfermeil, on a pu relever une réelle vitalité du modèle de PME familiale (dans le commerce de bouche ou le textile) porté par la communauté d'origine turque.

Mais un investissement important dans le système éducatif n'est pas garant d'une insertion professionnelle satisfaisante, générant en retour regret et amertume. Faute de réseaux, de connaissance du système scolaire et universitaire, certains jeunes s'engagent dans des cursus de formation inadaptés au marché du travail. Hassan, titulaire d'une maîtrise d'Administration et Gestion des Entreprises: "Toute cette énergie, tout ce temps perdu (...). Moi ce que je regrette, c'est que toute notre jeunesse, on nous a fait miroiter: "Fais des études, t'auras un bon boulot ", et finalité de l'histoire, c'est pas tout à fait ça». Redouane, titulaire d'une maîtrise d'AES (Administration des Entreprises et Société) "Pendant longtemps, j'ai pas travaillé. Mon cursus, j'ai bac+4. J'ai galéré pendant un an, même en cherchant dans l'intérim, dans des choses qui correspondaient pas à mon truc ». Au chômage ou cantonnés dans des métiers d'exécution alors qu'ils sont titulaires d'un bac +4 ou bac +5 , ils développent un fort ressentiment contre le système scolaire qui les a orientés de manière prédéterminée dans des voies sans avenir, faute de connaissance du marché du travail ou, selon eux, en raison de leur origine. Ce ressentiment s'exprime également contre la société française qu'ils estiment raciste et discriminatoire lors de leur recherche d'emploi : "C'est toujours mon faciès qui primait sur mes compétences ». Dévalorisés, ils ont reconstruit une image positive d'eux-mêmes au travers de la fréquentation de la mosquée et le projet, souvent évoqué dans les entretiens, de quitter la France qui les rejette, pour retourner dans leur pays d'origine ou s'installer dans un autre pays (Royaume-Uni, Moyen-Orient, pays du Golfe) : "Je vais être encore plus clair : je veux la [la France] quitter». L'islam leur permet une nouvelle construction identitaire, au risque parfois de la rupture avec la société française.

A l'autre bout de la chaîne, de nombreux jeunes sont en grande difficulté. Sortis très tôt du système scolaire, sans bagage éducatif ni culturel adapté, maîtrisant un français approximatif tant à l'oral qu'à l'écrit, ils ne disposent d'aucune clef pour comprendre le marché du travail et sont plongés dans une grande précarité. On retrouve cette caractéristique, mais pas exclusivement, dans nombre de familles originaires du Sahel. Parfois polygames, avec un taux de fécondité par femme très élevé, ces familles s'adaptent peu à leur société d'accueil. Les mères de famille ont le plus souvent des emplois harassants (femmes de ménage notamment) et les enfants sont soit délaissés, soit envoyés au pays pour y recevoir une éducation traditionnelle et échapper à la mauvaise influence de leur quartier. Ainsi Souleymane, né en France, a été envoyé au Mali à l'âge d'un an jusqu'à ses 18 ans, afin de recevoir une éducation "à la dure »; il cumule aujourd'hui les handicaps et se trouve dans une quasi impossibilité de s'insérer y compris au travers de petits boulots.

Le cœur du problème de Clichy-Montfermeil est l'emploi, comme dans l'ensemble de la société française, mais l'agglomération en représente un cas d'espèce, exacerbé par l'enclavement, les difficultés sociales et le capital éducatif et culturel inadapté d'une grande partie de sa jeunesse. Les parcours de galère rencontrés sont représentés par ceux qui, cumulant problèmes sociaux et absence de réseaux, parfois éjectés du système scolaire au sortir du collège ou mal orientés, quelquefois victimes de discriminations, ne parviennent pas à entrer sur le marché du travail et en viennent à retourner le stigmate dont ils souffrent en rejet radical de la France et des valeurs qui lui sont prêtées. 


\section{Chapitre 4 : La sécurité}

Le nom de l'agglomération de Clichy-Montfermeil reste lié dans l'esprit de la plupart des Français à la plus grande vague d'émeutes qu'ait connue l'histoire contemporaine de notre pays - entre la fin octobre et la mi-novembre 2005. Ces émeutes, auxquelles le gouvernement répondit en décrétant l'état d'urgence, donnèrent le sentiment que le modèle d'intégration français ne fonctionnait plus.

\section{Le « Grand Récit » des émeutes}

Déclenchées par l'électrocution de deux adolescents clichois, qui s'étaient réfugiés dans un transformateur EDF en fuyant la police, elles ont été relancées par l'explosion d'une grenade lacrymogène lancée par les forces de police, trois soirs plus tard, devant une mosquée où les fidèles étaient rassemblés en prière à l'occasion du Ramadan. Le "gazage de la mosquée " comme l'ont appelé nombre de participants à l'enquête, s'est avéré être le principal facteur déclenchant qui a propagé la révolte à Clichy-Montfermeil.

Comprendre comment s'articulent les deux causes que sont la mort de Ziyed et Bouna ainsi que le " gazage de la Mosquée », est l'une des clés d'interprétation de ces émeutes inédites en France par leur durée, leur répartition géographique et leur composition socio-ethnique. Les dysfonctionnements de la relation entre les banlieues populaires et la République, cristallisés par les émeutes de 2005, fédèrent une pluralité de causes : l'enclavement, le déficit éducatif ou encore l'inadéquation avec le marché du travail d'une partie importante de la population. Les émeutes ont constitué l'aboutissement d'un ensemble de processus qui manifestent au quotidien la rupture avec des normes sociales rejetées : de la dégradation du mobilier urbain au caillassage, en passant par les incendies de poubelles ou de véhicules, elles ont frappé également les équipements publics dont les habitants sont les premiers bénéficiaires, ainsi les écoles maternelles.

Ces manifestations ont nourrit le sentiment d'insécurité de nombre d'habitants et leur caractère autodestructeur a été largement commenté et interprété. Les conséquences des événements de 2005 ont également fait l'objet de lectures divergentes parmi les personnes interrogées: si pour certains le principal effet des émeutes est d'avoir déprécié l'image de l'agglomération et de ses habitants, d'autres relèvent une amélioration de la situation visible dans la Rénovation Urbaine et l'ouverture du commissariat, ainsi que l'émergence de nouvelles formes de politisation à travers la création d'associations qui ont favorisé la participation politique des jeunes.

\section{Le divorce entre police et population}

La genèse des émeutes de 2005 est emblématique du profond divorce entre police et population. Les deux adolescents suspectés d'avoir volé des matériaux de chantiers sont innocents, mais l'incompréhension est telle qu'ils préfèrent risquer leur vie pour se cacher plutôt que de clamer leur innocence. La police et les jeunes vivent dans deux mondes qui ne se comprennent plus. A entendre nombre d'enquêtés, la police est à la fois physiquement absente quand il faut assurer la protection des personnes et des biens dans les cités délaissées, et acharnée à nuire aux jeunes voire aux automobilistes victimes de contrôles « au faciès ».

Dans ce contexte, l'épisode du " gazage de la mosquée », quelles qu'en soient les circonstances exactes, a eu pour effet de dire symboliquement la profondeur du malaise, par-delà la seule police, entre les quartiers populaires à forte population d'origine immigrée et la République. Redouane, trentenaire d'origine marocaine, diplômé de l'enseignement supérieur et employé dans une association, constate : "normalement, c'est la police qui doit nous protéger, c'est elle qui protège les gens. [...] On doit tout faire nous-mêmes en fait. Même mon père, il me dit souvent: "on est abandonnés ici, il n'y a pas d'autorité publique ici. Les flics, ils tournent mais ils cherchent quoi ? Ils s'intéressent à qui? Aux ceintures de sécurité, aux gens qui ont fait un excès de vitesse". Je ne dis pas 
qu'il ne faut pas sanctionner ça mais il y a des priorités [...] Quand une personne nuit aux autres, on va voir ce qui se passe, on va l'interpeller. Les gens, ils sont $H 24$ devant les immeubles, ils fument leur shit. Les mères de famille, elles doivent passer, elles n'arrivent pas à passer. ”

Rares sont cependant les enquêtés qui souhaitent moins de présence policière. La moitié environ de l'échantillon s'est prononcée pour une présence policière accrue, répartie dans toutes les catégories d'âge, profession et origine, avec la quasi-totalité des personnes d'origine turque, et toutes celles d'origine pakistanaise. Pour la majorité des personnes interrogées, la qualité de la présence policière doit l'emporter sur sa quantité. Certains regrettent la disparition de la police de proximité comme telle et beaucoup estiment que la visibilité des forces de l'ordre dans le paysage quotidien, leur fonction dissuasive, doivent l'emporter sur les opérations coup de poing maladroites, alors que d'autres estiment qu'elles doivent d'abord se faire respecter pour s'imposer face à la délinquance, aux dealers de drogue pour commencer, et aux pratiques d'incivilité quotidienne.

La plupart de ceux qui appellent de leurs vœux un retour de la police mettent l'accent sur la prévention et la dissuasion. Rabia, trentenaire d'origine turque, diplômée en droit, accueille favorablement l'arrivée du commissariat, mais sous conditions : "Il y a un commissariat qui va s'ouvrir bientôt au mois de septembre, peut-être que ce sera mieux. Mais j'aimerais mieux qu'ils partent à la recherche des jeunes, pour qu'ils se fassent connaître et que les jeunes n'aient pas peur des policiers, ça, ça sera plus intéressant ». Hassan, trentenaire marocain diplômé aspire quant à lui à : " une police, justement, qui sait dialoguer. C'est une police expérimentée, qui connaît le terrain, qui sait comment prendre le problème. C'est pas une police qui vient, boum, coups de matraque. C'est pas une police répressive, spécialement, que j'attends. Y a besoin de répression, certes, mais vous avez besoin de prévention avant la répression. »

\section{Un sentiment d'insécurité contrasté}

Paradoxalement, les deux tiers des personnes interrogées disent se sentir en sécurité, particulièrement deux catégories de l'échantillon : les jeunes hommes d'origine maghrébine et africaine résidents des grands ensembles, mais aussi les habitants des pavillons de l'agglomération et des communes voisines qui ont vécu les émeutes comme un phénomène largement extérieur. Ce sentiment est souvent structuré par un phénomène d'appartenance à la communauté locale et d'interconnaissance. Les personnes interrogées sont bien conscientes de la fragilité de cette sécurité, qui repose sur un rapport de force qu'il faut constamment renouveler et suppose le respect d'un certain nombre de codes. La sécurité des personnes ne signifie cependant pas que le reste de la vie quotidienne soit sécurisé : l'occupation de cages d'escaliers et de halls d'immeubles par des bandes de jeunes, le bruit, les dégradations, le trafic de drogue empoisonnent l'existence et contribuent à un ressentiment fréquemment exprimé contre la police. En revanche, les habitants qui ne se sentent pas en sécurité, parmi lequel une majorité de femmes, et plusieurs membres de groupes minoritaires, nourrit ses peurs des groupes majoritaires - principalement les jeunes d'origine maghrébine ou africaine - par le fait qu'ils ne leur appartiennent pas et ne sont pas protégés par des codes ou une subculture commune. Ces habitants ont ressenti les émeutes comme une violence dont ils étaient les victimes directes ou indirectes, et dont les jeunes portaient la responsabilité.

Contrairement à ce qu'auraient pu imaginer ceux qui voient dans les quartiers populaires des banlieues des zones de non-droit où une population communautarisée serait arc-boutée contre les ingérences de l'État, l'ouverture du commissariat à Clichy-Montfermeil a suscité des attentes importantes chez les habitants, qui y ont vu l'un des éléments du retour à la normalité, le signe qu'ils ne seraient plus abandonnés des pouvoirs publics et pourraient mieux jouir de leurs droits de citoyens - la sécurité au premier chef. L'intégration à la vie locale du nouveau commissariat a transformé dès les premiers mois le rapport à la police. De sa capacité à tisser un lien avec la population et avec les autres instances et institutions présentes sur le territoire dépendra en partie la résorption de la coupure entre la République et sa banlieue dans l'agglomération de Clichy-Montfermeil. La restauration de la sécurité dans les quartiers sensibles de l'agglomération de Clichy-Montfermeil ne peut se réduire à une politique de répression : celle-ci n'a d'efficacité sociale que si elle se déploie en parallèle avec 
prévention et dissuasion, largement délaissées dans ces territoires. Et la prévention ne peut advenir si la majorité de la population concernée n'adhère pas aux valeurs de la société ambiante, telles qu'elles se traduisent au concret dans l'environnement urbain, éducatif et l'accès au marché au travail. C'est l'interaction de ces divers domaines avec l'amélioration de la sécurité ainsi que l'acceptation de ses conditions qui tisse la réinsertion de ces quartiers dans la République. 


\section{Chapitre 5 : La politique}

Quel rapport les banlieues entretiennent-elles avec le politique ? L'étude du cas de Clichy et de Montfermeil, autrefois communistes et devenues respectivement socialiste et de droite permet de répondre de façon nuancée à cette question. Ces deux communes pèsent environ 30000 habitants chacune et comptent un tiers de leur population inscrite sur les listes électorales, un taux deux fois moins élevé que celui constaté à l'échelle nationale, en dépit du flux massif d'inscriptions sur les listes électorales consécutif aux événements de 2005. Ce décalage entre pays réel et pays légal s'explique en partie par la forte proportion d'étrangers et de mineurs dans ces zones urbaines sensibles.

\section{Les aléas de la participation politique}

Parmi les personnes majeures de nationalité française de l'échantillon, $80 \%$ ont déclaré qu'elles votaient ou avaient voté et à la question "La politique est-elle importante pour vous ? ", 55\% des enquêtés répondent positivement. Les jeunes nés en France, surtout lorsqu'ils sont diplômés et actifs, revendiquent une participation de plain-pied à la vie politique. Au lendemain des événements de 2005, Clichy-Montfermeil fut à l'avant-garde d'une forte progression des inscriptions de jeunes Français issus de l'immigration sur les listes électorales, grâce à des associations civiques et marquées à gauche comme ACELEFEU !, mouvement de mobilisation dont l'acronyme (assez le feu !) pour "Action Collectif Liberté Egalité Fraternité Ensemble», disait bien la volonté de traduire des émeutes, rebaptisées « révoltes sociales » en action politique. Le collectif appela à voter pour Ségolène Royal au second tour de l'élection présidentielle de 2007. Si celle-ci y recueillit plus de $60 \%$ des voix à Clichy, tandis que le taux de participation y culminait à $82 \%$, son échec national se traduisit dès les législatives suivantes par une forte démobilisation et aux élections régionales de 2010, Clichy-sousBois figure parmi les communes les plus abstentionnistes de France avec 31,3\% de votants au second tour. Toutefois, les espoirs suscités par la forte mobilisation de la jeunesse restent vifs et l'enquête a permis de montrer que les banlieues sont loin d'être des déserts politiques.

Sans surprise, l'intérêt pour la politique est étroitement corrélé aux variables sociologiques traditionnelles, au premier rang desquelles on trouve le niveau d'éducation. Parmi ceux qui manifestent le moins d'intérêt et ne votent pas, la faiblesse du niveau culturel, la non détention de la nationalité française et l'origine sahélienne reviennent fréquemment. Bintou, animatrice de 23 ans d'origine malienne, déclare : " ne rien comprendre à la politique ». Pour Isabelle, gardienne d'immeuble quadragénaire, la politique fait partie «des choses qui la dépassent », alors que Vinz, 25 ans, Antillais issu d'une famille monoparentale, sans emploi et ayant arrêté ses études au lycée affirme : «Moi, je n'ai pas de pouvoir, mon vote il sert à rien! ».

L'enquête a révélé une forte dépréciation des responsables politiques au niveau national. Si les entretiens confirment un "déficit d'image » marqué des figures de l'actuelle majorité, notamment le Président de la République, dans les banlieues, les hommes et femmes politiques de gauche ne sont pas épargnés et dans l'ensemble, les habitants de Clichy-Montfermeil, toutes citoyennetés confondues, paraissent en phase avec l'ensemble de la population française. Peu de voix s'élèvent pour marquer de la confiance au personnel politique national, d'autant plus décrié qu'il apparait comme lointain. Pour Roland, septuagénaire et ouvrier retraité : «Le pouvoir en place à l'heure actuelle, c'est celui de ceux qui sont riches et qui veulent le rester, donc ils n'ont pas trop d'intérêt à changer le système économique actuel. » Salif, 20 ans, d'origine malienne et sans emploi, ayant quitté les études au lycée, justifie son retrait de toute activité politique, bien qu'il en perçoive l'importance, à cause du dégoût que lui inspirent les politiciens : «La politique, c'est la politique, après je ne m'en mêle pas! (...) Oui, c'est important. Dans un pays, il ne peut pas ne pas y avoir de politique. Mais pour moi, les politiciens, ce sont des voleurs, des menteurs, ce sont des tricheurs, voilà!» Seul le maire, souvent l'unique figure politique visible et accessible, fait l'objet d'appréciations, positives ou négatives, d'un autre ordre, car fondées sur un bilan concret et tangible. 


\section{Prégnance de la religion et défi de l'emploi}

L'intérêt pour le politique revêt à Clichy et Montfermeil des formes particulières. L'enquête révèle une imbrication forte du politique et du religieux. L'importance des populations de confession musulmane et le potentiel électoral qu'elles représentent, et dont aucun responsable politique ne peut prendre le risque de laisser bénéficier uniquement l'adversaire, a fait de la construction des mosquées une thématique que les programmes de campagne ne peuvent plus oublier et de la tournée des salles de prières un passage obligé des candidats aux élections.

Dans les thèmes considérés comme prioritaires par les enquêtés, la question de l'emploi, préoccupation majeure des Français à l'échelle nationale, revêt une tout autre ampleur sur un territoire où le taux de chômage représente le double de la moyenne nationale. Cette question est identifiée largement en tête des problèmes politiques, loin devant les questions d'intégration ou de sécurité. Ainsi, Rabia, trentenaire d'origine turque qui a étudié le droit, déclare que «franchement, pour moi le vrai problème, ce n'est pas la sécurité en France, c'est l'emploi, l'emploi pour les jeunes notamment, les jeunes qui ne savent pas quoi faire de leur journée. » Dans l'ensemble, on note une forte défiance envers la manière dont la question de la sécurité est abordée par les responsables politiques, la majorité des personnes interrogées jugeant que ce thème est largement instrumentalisé à des fins électoralistes. C'est ainsi que la proposition qui avait été faite en juin 2010 de déchoir de leur nationalité les criminels naturalisés ayant abattu un policier est majoritairement rejetée par les enquêtés. Ces derniers y voient une volonté de créer une hiérarchie entre Français, renforçant le sentiment de stigmatisation des populations d'origine étrangère.

\section{Etre français}

Au cœur du problème de l'accès au politique se trouve la question de la nationalité française, avec l'ensemble de ses connotations culturelles, économiques et politiques. Si pendant longtemps, l'acquisition de la nationalité française a représenté, pour les primo-arrivants, un cheminement malaisé, apparaissant souvent comme une absurdité ou une aberration voire comme un reniement ou, pire encore, une apostasie, les immigrés de première génération l'acquièrent de plus en plus et votent.

Les réponses à la question "Pour vous, être français, ça veut dire quoi ? " révèlent qu'il n'est pas possible de dissocier la question de la participation politique de celle de la définition de l'identité. Si $70 \%$ de l'échantillon interrogé est composé de personnes de nationalité française dont au moins un des deux parents est né à l'étranger, les postures adoptées vont de l'adhésion aux valeurs et aux idéaux républicains (à l'instar de Pierre, ingénieur trentenaire d'origine turque, pour qui être français «c'est un certain nombre de valeurs, la tolérance, l'égalité, la laïcité, la fraternité ») jusqu'à la volonté de quitter la France, avec souvent l'expression d'une frustration de ne pas véritablement être reconnu comme français par ses compatriotes « de souche ». L'impossibilité de se sentir français dans le regard de l'autre majoritaire, exacerbée par la notion d'imprescriptibilité raciale - notamment chez certains jeunes d'origine sahélienne qui, en dépit de leur nationalité, ne se sentent pas français - tend à limiter la participation politique ou à accroître le sentiment d'amertume vis-à-vis du système politique comme le rapporte Hassan, trentenaire d'origine marocaine et vivant d'emplois précaires en dépit de ses diplôme universitaires : "Honnêtement, lorsqu'on quitte, on va dire le quartier, les coins qu'on connaît, y a rien qui fait de nous des Français. Le regard des autres ne fait pas de nous des Français, les questions des autres ne font pas de nous des Français... ».

L'enquête conduite auprès des habitants de Clichy et de Montfermeil révèle finalement que ces populations ne sont pas perdues pour le politique, mais que cette politisation exige des conditions et revêt des formes qui sont propres à ce territoire. La question qui se pose alors est celle des possibilités d'actualisation de cette politisation sous des formes institutionnelles et conventionnelles, actualisation d'autant plus problématique que la première génération d'élus locaux issus de l'immigration peine à se faire reconnaître par les appareils des grands partis et à parvenir à des positions de forte responsabilité 
issues du suffrage universel. Ces élus, parfois instrumentalisés pour obtenir du «vote ethnique » risquent de finir par se spécialiser, devenant les porteurs d'un vote communautariste qui, pour l'heure, n'est pas apparu prégnant à travers l'enquête. Il semblerait dès lors pertinent que les grands partis favorisent l'accès à des positions éligibles de premier plan pour ces jeunes issus des vagues d'immigration récentes. 


\section{Chapitre 6 : La religion}

Les résultats de l'enquête sur le rapport au religieux font une large place à l'islam, puisqu'il s'agit de la religion des deux tiers des enquêtés. La relation du territoire de Clichy-Montfermeil à l'islam est marquée par une piété qui semble exacerbée par les circonstances particulières que connaît l'agglomération, allant de l'enclavement géographique à l'adversité sociale. L'analyse des résultats revêt dès lors moins une dimension représentative qu'une valeur emblématique de la place du religieux au sein de banlieues : en effet, il importe de toujours considérer l'écart entre ces résultats et les différents sondages sur les pratiques de l'islam sur l'ensemble du territoire français.

\section{Extension de la notion de halal}

Comparativement aux réponses obtenues un quart de siècle auparavant dans les Banlieues de l'islam (1987) de Gilles Kepel, l'enquête montre une véritable explosion du marché du halal et l'extension de la notion. Les résultats à la question «respectez-vous le halal ? » mettent en lumière la complexité des différentes acceptions de ce mot, qui dans sa définition la plus restrictive ne recouvre que la dimension de l'interdit alimentaire, mais peut aussi s'étendre à un code de conduite, une expression des normes et valeurs dominantes, en séparant le «halal» du " haram », le licite de l'illicite, dans de nombreux domaines depuis le registre de l'intime jusqu'à la vie en société. Ainsi, pour Khadidja, mère de famille d'origine marocaine, "Le halal, c'est de ne pas faire entrer de choses volées chez soi. Le halal, c'est faire comprendre à ses enfants qu'ils doivent être honnêtes. Le halal, c'est pas mélanger de l'argent qui est gagné à la sueur de son front avec un euro qui est malsain. Ça c'est halal. Le halal, c'est être fidèle à son mari. Le halal, c'est être fidèle à ses enfants, à ses amis. C'est très vaste..."

La question du mariage entre dans ce champ : la majorité des enquêtés souhaite que leurs enfants se marient avec une personne pratiquant le même culte. Cette préférence pour le mariage endogame revient également dans les réponses des chrétiens d'Orient, qui y voient la seule manière de préserver l'existence de leur communauté. A l'échelle de Clichy-Montfermeil, le mariage est donc essentiellement considéré comme un moyen de renforcement de la cohésion communautaire. Si le halal contrôle le lien entre l'intime et le social, c'est la mosquée qui assure l'interface avec la communauté, définie par le lien religieux spécifique entre les croyants et la société. C'est dans le courant des années 1980 que les premières structurations de l'islam local voient le jour, essentiellement au sein de locaux situés au rez-de-chaussée de grands ensembles dégradés. ClichyMontfermeil compte aujourd'hui une dizaine de salles de prière, d'une capacité d'accueil de 200 à 1 700 places, pour environ 12000 fidèles. La gestion de ces espaces est assurée par des associations cultuelles, leur fréquentation et leur orientation étant fortement influencée par l'origine nationale du fondateur ou du président de l'association.

\section{Le tabligh et la figure de l'imam : les deux pôles majeurs du culte musulman}

L'offre d'islam à Clichy-Montfermeil est structurée autour de deux pôles majeurs : l'un est lié au mouvement tabligh («propagation de l'islam »), association piétiste et prosélyte, qui se caractérise par une resocialisation islamique ciblée vers les milieux les plus fragiles. Arrivés dans le quartier de Clichy-Montfermeil dans les années 1980, alors que s'installaient chômage de masse et problèmes de drogue, ces prêcheurs ont construit leur légitimité sociale en assurant une régénération morale des jeunes en perdition, autour d'une pratique rigoriste des préceptes de l'islam. Pour certains, leur action a permis l'éradication des drogues les plus dures dans des quartiers où l'Etat s'était désinvesti. La socialisation par le tabligh a touché beaucoup de jeunes et certains anciens pratiquants, ayant adopté depuis une attitude plus distancée, en ont gardé une éthique et une rigueur morale les conduisant souvent à un engagement laïc dans la vie associative ou la vie politique locale.

Le deuxième pôle est lié à la figure de l'imam Dhaou Meskine, qui officia pendant 25 ans dans la mosquée Stamu à Clichy. Il participa au lancement de l'UOIF (Union des Organisations islamiques de 
France), fut un temps l'interlocuteur privilégié de Nicolas Sarkozy dans la création du Conseil Français du Culte Musulman (CFCM) et fondateur du premier collège lycée musulman de France, situé à Aubervilliers. Dhaou Meskine se présente comme un modéré, en comparaison aux membres du tabligh et aux salafistes, présents depuis le milieu des années 1990, mais ne disposant d'aucun lieu de culte et dont l'influence reste très limitée.

\section{Le projet de Grande Mosquée et la naissance d'un « lobby musulman »}

C'est dans ce contexte complexe qu'a pris naissance le projet de construire une Grande Mosquée dans l'agglomération, emblématique des difficultés auxquelles se heurte le processus d'harmonisation entre autorités locales et associations cultuelles pour favoriser l'exercice légitime du culte musulman dans le respect des lois de la République. Créée en 2003, l'Association Culturelle de la Grande Mosquée de Clichy Montfermeil (ACGMC), regroupe huit associations et sa mission revient pour certains à « fédérer l'infédérable ». Alors que l'ouverture de la Grande Mosquée était prévue en février 2011, le chantier ne parvient pas à dépasser le stade du terrassement, du fait de deux blocages majeurs : l'un concerne le conflit opposant l'entrepreneur à l'ACGMC du fait des sommes non versées (les coûts nécessaires à la finalisation des travaux étant estimés entre 5 et 7 millions d'euros), l'autre concerne une lutte d'influence entre les différentes communautés d'origine (turque, marocaine, algérienne) et entre associations proches de divers partis politiques français au sein de l'ACGCM. Au niveau local, la construction de la Grande Mosquée constitue un enjeu électoral majeur : il s'agit à la fois pour les élus de satisfaire les attentes d'un électorat musulman «flottant» récemment inscrit sur les listes, mais ne participant pas aux élections de manière systématique, et de ne pas heurter l'électorat plus traditionnel, craignant « l'islamisation de la France », comme l'illustrent certaines prises de position sur des blogs identitaires.

C'est dans ce contexte que s'est construit le premier «lobby musulman » de France : l'Union des Associations Musulmanes (UAM 93) dont l'objectif est de mobiliser le vote communautaire afin de peser sur les candidats aux élections locales autour d'enjeux islamiques, dont le premier est la construction de mosquées pour les 600000 musulmans que compterait la Seine-Saint-Denis. Les relations complexes entre islam et politique locale sont à analyser au regard de la perception des musulmans du regard des Français sur leur communauté. On note chez les primo-migrants des réponses nuancées puisque $14 \%$ estiment que «les Français nous voient mal » contre $44 \%$ des jeunes interrogés nés en France. Pour Moustapha, étudiant à Sciences Po d'origine marocaine, "ils nous voient de moins en moins bien et, depuis que les langues ont commencé à se délier, on a de moins en moins de retenue. Bien sûr, ça ne concerne pas tous les Français, j'ose espérer que ça ne concerne qu'une minorité, mais je crois qu'aujourd'hui, il y a beaucoup d'incompréhension face à l'islam, beaucoup de clichés, beaucoup de fausses idées. On peut vraiment dire que l'islam est de loin, la religion, la moins respectée dans les médias. »

Alors que les chrétiens interrogés se sentent majoritairement respectés, les musulmans estiment être stigmatisés et constatent un déni de leur statut de victime, contrairement à l'attention portée selon eux à la communauté juive. Ce rapport de miroir ambivalent de la communauté musulmane vis-à-vis de la communauté juive s'est forgé au sein d'un imaginaire complexe, allant du "gazage de la Mosquée Bilal » à l'identification aux Palestiniens de Gaza opprimés par Israël, en passant par l'édification du concept d'islamophobie construit comme un parallèle de l'antisémitisme. Certaines de ces comparaisons témoignent d'un respect pour une stratégie efficiente, alliant une capacité à représenter ses intérêts sur la scène politique et à maintenir une forte cohésion communautaire. A cet égard la communauté juive aurait réussi à éviter la dilution dans la société laïque, là où les chrétiens ont partiellement échoué et peut devenir un modèle pour l'émergence d'un lobby musulman au niveau national.

De manière plus globale, le blocage de la construction de la Grande Mosquée de Clichy-Montfermeil est emblématique des difficultés liées à la construction de nouveaux lieux de cultes en France, du fait de la loi de 1905 sur la séparation des Eglises et de l'Etat. Les pouvoirs publics n'étant pas en mesure de participer au financement de ces lieux, leur construction repose essentiellement sur les sommes 
collectées par les fidèles. Alors que le nombre de mosquées est passé de 1536 en 2000 à 2101 en 2010 - soit une ouverture de mosquée par semaine en dix ans sur le territoire métropolitain -, l'absence d'évolution législative, malgré les différentes propositions émises notamment par la commission Michalon, en vue d'une harmonisation dans le respect des lois de la laïcité, rend très complexe l'intégration harmonieuse de ces lieux dans l'environnement urbain et social. Or la sociologie des nouveaux fidèles musulmans étant essentiellement constituée de personnes à faibles revenus, ce financement n'est pas rendu possible sans l'apport de financements étrangers, auquel l'Etat n'est pas favorable. Ces contradictions se retrouvent dans les propos des fidèles, qui ne souhaitent pas a priori que l'Etat interfère dans les questions relatives à l'islam, mais qui attendent cependant de lui de débloquer les situations inextricables. 


\section{Institut Montaigne et cohésion sociale : une longue histoire}

Ecole, logement ou entreprise, c'est depuis 2004 que l'Institut Montaigne s'intéresse aux " oubliés de l'égalité des chances ». Qu'il s'agisse de donner aux entreprises les couleurs de la France, de vaincre l'échec à l'école primaire, de réfléchir au logement social pour que tous aient un toit, l'Institut Montaigne a formulé des propositions pour plus de justice sociale. C'est dans la lignée de ces travaux que l'Institut Montaigne mène une enquête sur l'agglomération de Clichy-Montfermeil depuis l'été 2010.

- Les oubliés de l'égalité des chances. Rapport, janvier 2004.

- Ni quotas, ni indifférence : l'entreprise et l'égalité positive. Note, octobre 2004.

- Des entreprises aux couleurs de la France. Rapport, novembre 2004.

- Ouvrir les grandes écoles à la diversité. Rapport, janvier 2006.

- Ecoles primaires en ZEP : faire plus et différemment. Note, septembre 2006.

- Sortir de l'immobilité sociale à la française. Note, novembre 2006.

-HLM, parc privé. Deux pistes pour que tous aient un toit. Note, juin 2008.

-Engager le citoyen dans la vie associative. Rapport, novembre 2008.

- Ouvrir la politique à la diversité. Note, janvier 2009.

- Comment déghettoïser les « quartiers », un exemple concret : les Bosquets à Montfermeil. Note, juin 2009.

- Qu'est ce qu'être Français? Recueil de contributions, novembre 2009.

- Vaincre l'échec à l'école primaire. Rapport, avril 2010.

- Quelle place pour les minorités visibles? Retour sur les élections régionales de mars 2010. Note, septembre 2010.

- 15 propositions pour l'emploi des jeunes et des seniors. Rapport, septembre 2010.

Pour nous contacter :

Michaël Cheylan, directeur des affaires publiques de l'Institut Montaigne : mcheylan@institutmontaigne.org - 01.58.18.39.26 